\title{
CrimRxiv
}

\section{Review 2 of "Socialization across the three stages of the Correctional Service of Canada's correctional officer training program: An ethnographic study"}

Helen Arnold Published on: Jul 13, 2021 License: Creative Commons Attribution 4.0 International License (CC-BY 4.0). 
Vote: Publish pending minor changes

[For votes to count, referees must reasonably explain why they voted as they did. Thus, please explain your vote. If you voted to publish pending minor changes, specify each change, why it is needed, and, possibly, how it should/could be done.]

This paper makes an important and unique contribution to the body of literature on correctional officers offering insight into the process of organizational socialization across stages of the correctional officer training program and outcomes in terms of organisational commitment and the perpetuation of correctional officer culture. The paper clearly defines the key concepts and draws specifically on Van Maanen and Schein's (1977) theory of organizational socialization which lends the paper a clear focus and analytical lens; this theory and the component tactics are explained well and made easy to understand. The paper refers to, but does not over-rely on, previous (and notably limited) criminological studies in this area.

The paper is competently executed in that it is written in an accessible and clear fashion and is well structured and organized. The topic is explained well, and the study not only addresses a gap in the literature but also addresses a methodological gap - in relation to method, there is potential for the value and uniqueness of the study to be more strongly emphasized; that the researcher undertook the CTP herself is both commendable and rare within prisons research and offers a very privileged and distinctive point of observation, reflection, and analysis. The structure and demands of the correctional officer training program is made clear within the results section, and, whilst the ethical considerations and practices are not discussed in depth, my view is that this would detract from the essence of this article and would more usefully be published in a separate paper. Analysis is appropriately thematic and is based on the authors own experience as a correctional officer recruit and interpretation of selfgenerated data in the form of field and training notes. Interpretation and explication of the data in the form of results is good in that original content is presented, for example in the notion of anticipatory socialization. The distinction and relationship between collective and individual emphasis in socialization is captured well and forms the bulk of the study findings where the author proposes that, contrary to accepted knowledge and previous academic study, camaraderie is a contested and caveated element of correctional officer culture that has its roots, and is taught in, initial training. The concept of caveated comradery signals the original contribution of the 
paper and has much validity in helping to understand more deeply the work of correctional/prison officers and workplace behaviours and stress. Finally, the paper makes appropriate and meaningful recommendations for areas of future research that align with the study findings and limitations and show a logical direction of travel.

Overall, this paper is very well thought out, academically sound and makes an important contribution to the field.

Minor Changes:

1. There are some errors in spelling and grammar that are resolvable by a thorough proofread.

2. At the end of the second paragraph under the 'Organizational Socialization' section the link between 'diverse cognitive orientations' and the 'for instance' provided should be made clearer.

3. At the end of the third paragraph under the 'Organizational Socialization' section it may be useful to clarify (the difference between) serial and disjunctive and investiture and divestiture in a few words.

4. The relevance of culture to this study should be made more evident; prison officer culture is a significant topic of study and is difficult to do justice to in summary; a focus on solidarity (rather than attitudes) as a component of culture would provide a better 'fit' given the focus of this paper.

5. The inter-relationship between socialization, commitment and culture could be made clearer; whilst the three terms are well defined there could be more coherence with the study findings.

6. Rename 'Testing' to 'Stress' to be consistent with the stated elements for discussion in the section 'Anticipatory Socialization', or label iii) testing instead of 'stress'.

7. It would be interesting to know how many modules there were in total to be able to contextualize how many involved testing.

8. Some sections of the paper would benefit from a re-read with a view to presenting the key findings and ideas more succinctly - there is some slight repetition between the findings and the discussion, some inequality in length between the findings section, which could be reduced to leave the more detailed explanation to the discussion. 
